\title{
New micropattern gas detectors for the endcap muon system of the CMS experiment at the high-luminosity LHC
}

\author{
Cesare Calabria on behalf of the CMS Muon Group* \\ Universitá \& INFN Bari \\ E-mail: cesare.calabria@ba.infn.it
}

\begin{abstract}
For the era of the high-luminosity LHC [1], new detectors are planned to enhance the performance of the endcap muon system of the CMS detector[2]. We report on two types of these detectors that will be installed during the third long shutdown (LS3) of the LHC. In the pseudo-rapidity region $1.6<|\eta|<2.4$, new triple-foil large-area Gaseous Electron Multiplier (GEM)[3] detectors will be installed in the third of five detector stations in each endcap, the first station being closest to the interaction point. These GEM detectors are in addition to ones that will have already been installed in the second station during LS2. We present a design for the third station detectors that must cover a larger geometrical area than those in the second station, while maintaining good performance for efficiency and spatial resolution. A new innermost (first) detector station will be installed in the endcaps to extend the range of muon identification up to about $|\eta|=$ 3.0. We describe the geometrical constraints and particle fluxes at the first station. The detector technologies under consideration include Fast-Timing Micropattern (FTM)[4] structures that can tolerate large particle fluxes and provide good time resolution. FTM detectors employ multiple layers of resistive-coated kapton foils with either hole or mesh electron multiplication structures. We report on the performance of prototype FTM devices for efficiency, space and time resolution measured using X-rays, cosmic-ray muons, and extracted high-energy particle beams.
\end{abstract}

38th International Conference on High Energy Physics

3-10 August 2016

Chicago, USA

\footnotetext{
* Speaker.
} 


\section{CMS Muon System Upgrade towards HL-LHC}

The CMS muon system is designed to provide robust, redundant and fast identification of the muons traversing the system, in addition to trigger capabilities and momentum measurement. Precision measurements are provided by Drift Tubes (DT) in the barrel, covering acceptances up to $|\eta|<1.2$ and Cathode Strip Chambers (CSC) in the endcaps covering $1.0<|\eta|<2.4$. Resistive Plate Chambers (RPC) cover the region up to $|\eta|<1.6$. The region $|\eta|>1.6$ is only instrumented with CSC. One of the main goals during the upgrade phases will be to restore the originally foreseen redundancy in the forward region beyond $|\eta|>1.6$ based on modern, high-resolution and fast gas detectors capable of fully exploiting the increased LHC performance and sustaining reliable operation for the next decades. Therefore such detectors have to satisfy a high rate capability, $250 \mathrm{~Hz} / \mathrm{cm}^{2}-100^{\prime} \mathrm{s} \mathrm{kHz} / \mathrm{cm}^{2}$ (Fig. 1), a good time resolution for triggering, and a good spatial resolution, $O(100 \mu \mathrm{m})$, for tracking. The current CMS RPC design is not able to sustain the high rates expected during the next phases of LHC.
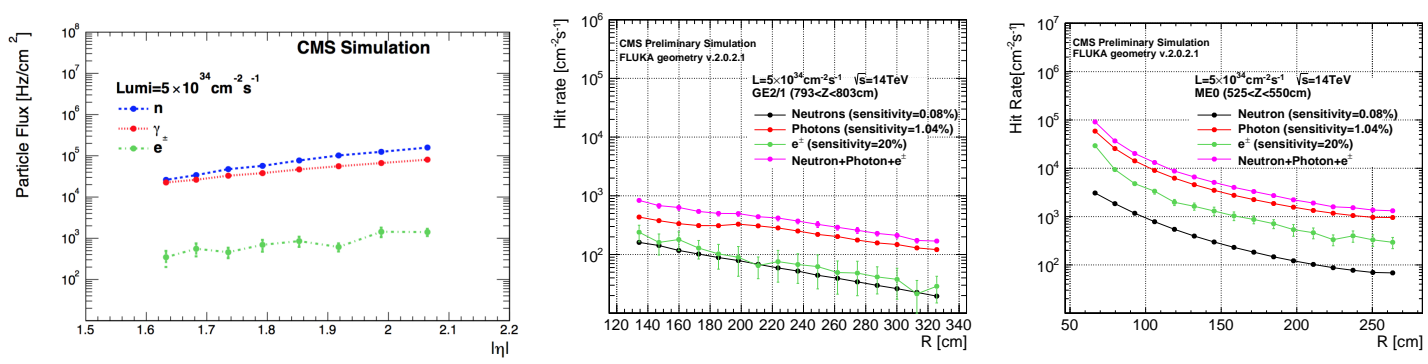

Figure 1: Background rates simulated with FLUKA+GEANT4 for GE1/1 (right), GE2/1 (center) and ME0 (left). Rates expected in each chamber are normalized to the instantaneous luminosity of $5 \times 10^{34} \mathrm{~Hz} / \mathrm{cm}^{2}$.

\section{The Forward Muon System enhancement}

The baseline of the CMS Muon System Upgrade (Fig. 2) is the installation of 36 doublelayered triple-GEM chambers in front of the ME1/1 station during the LHC second Long Shutdown (LS2), called the GE1/1 system [5]. The chambers will provide full coverage in $\phi$ and $1.55<|\eta|<$ 2.18 in pseudo-rapidity. The odd-numbered GE1/1 chambers will be slightly larger to maximize coverage in $|\eta|$. The station 2 upgrade (GE2/1) consists of installing two rings of double-layered triple-GEM chambers covering up to $1.6<|\eta|<2.45$ during the third Long Shutdwon (LS3). Each GE2/1 chamber spans about 20 degrees in $\phi$. Moreover a muon near-tagger (ME0) with 18 sixlayered triple-GEMs, with each chamber providing coverage of 20 degrees in $\phi$ and $2.0<|\eta|<3.0$ in pseudo-rapidity, behind the future shortened hadron calorimeter (after LS3)[6].

The triple-GEM has a trapezoidal shaped active area of $990 \times(220-455) \mathrm{mm}^{2}$ with a 3/1/2/1 $\mathrm{mm}$ drift/transfer-1/transfer-2/induction field gap configuration. The readout is segmented in up to $8 \times 3(\eta, \phi)$ partitions with 128 radial strips each, with a strip pitch ranging between 0.6-1.2 $\mathrm{mm}[5]$. The GE2/1 chambers will be similar to the GE1/1 basic unit, albeit with dimensions of about $1.2 \times 0.8 \mathrm{~m}^{2}$, so covering a much larger surface. GE2/1 has to be very thin (only $81 \mathrm{~mm}$ width) to fit the available space in front of ME2/1. Moreover, in order to cover such a big area, 2-4 
GEM foils have to be spliced together to build a chamber and this means having four independent detectors to be coupled together. The R\&D for GE2/1 is still ongoing. Although also the R\&D for ME0 is still ongoing, it is clear a high granularity detector is needed to assign $p_{T}$ through $\Delta \phi$ measurement and to improve the pile-up rejection. A multi-layered structure is necessary to improve the local muon reconstruction and to discriminate muons against neutrons. Precision timing is also required to reduce the in-time PU. Moreover ME0 has to fit the available space (20.5 $\mathrm{cm}$ ) behind the future endcap calorimeter.

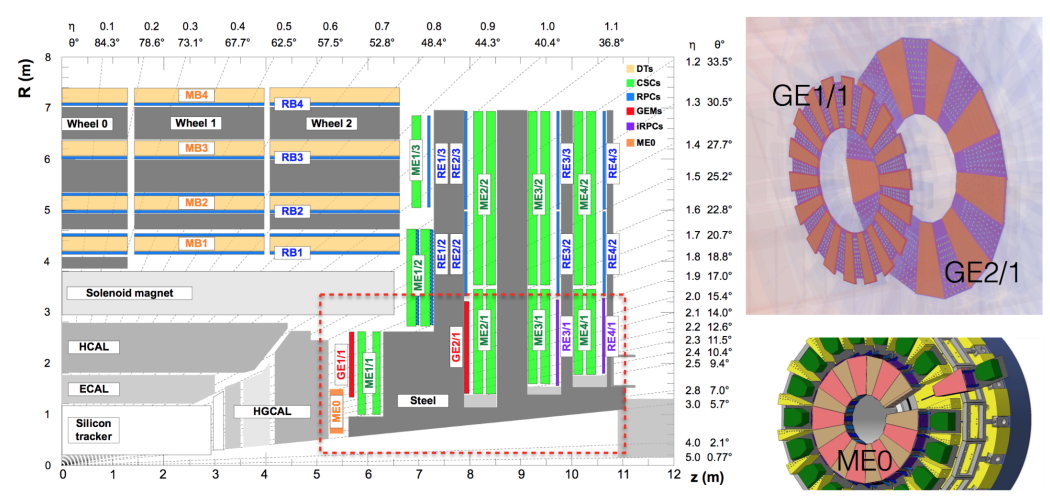

Figure 2: Transverse section of CMS showing the Muon system with DTs, CSCs and RPCs. The proposed GEM stations, GE1/1, GE2/1 and ME0 are given by the red and orange respectively.

\section{GEM Detector optimization $\&$ performance}

Six GEM prototypes have been developed since 2009. The first large GEM prototype, GE1/1I, had all components glued together, had spacers and 8 readout sectors. This number of spacers was increased to 24 in GE1/1-II. GE1/1-III was the first type to make use of the manual stretching technique (NS2), that allows to adjust the tension accordingly. This technique introduces a new way to stretch the foils and assembly a chamber without spacer and glue, reducing assembly time and allowing a fast and easy repair of broken chambers. In addition, the distances of the drift, transfer and induction region were finalized. GE1/1-IV was the first prototype to be fully mechanically constructed and produced at various production sites around the world. The GE1/1-V have an optimized coverage in $\eta$ and $\phi$ and have short and long types. The final version GE1/1-VI has the same features as the $\mathrm{V}$ prototype with an optimized coverage in $R$. Triple-GEM technology perfectly meets the requirements imposed by the HL-LHC providing high rate capability (up to $\left.O\left(M H z / \mathrm{cm}^{2}\right)\right)$, good time (4ns) and spatial resolution $(268 \mu \mathrm{m})[5]$.

\section{The next MPGD architecture}

The structure of the first prototype of FTM detector is described in Fig. 3 left. It is composed of two independent drift-amplification stages: each amplification region is based on a pair of polyimide foils, i.e. kapton, stacked due to the electrostatic force induced by the polarization of the foils. The first foil, perforated with inverted truncated-cone-shaped holes is a $50 \mu \mathrm{m}$ thick polyimide foil coated with diamond-like carbon (DLC) technique, to reach a specific surface resistance 
of up to $800 M \Omega / \square$. The second foil is $25 \mu \mathrm{m}$ with a resistivity of $2 M \Omega / \square$. The drift volumes are $250 \mu \mathrm{m}$ thick, with planarity ensured by overlay pillars. The first prototype of Fast Timing Micropattern detector was tested and proved using X-rays to have a linear response to the rate, to be electrically transparent and its time resolution was measured (using high-energy pion and muon beams) to be of the order of 1.5-2.5 ns with $\mathrm{Ar} / \mathrm{CO} 2$ 70/30 gas mixture (Fig. 3, center and right plots)[7]. The detector is being considered for applications in high energy physics experiments, including the CMS muon system. The $\mathrm{R} \& \mathrm{D}$ is ongoing with the design of a new prototype, fully PCB-based, with at least 4 independent stages.
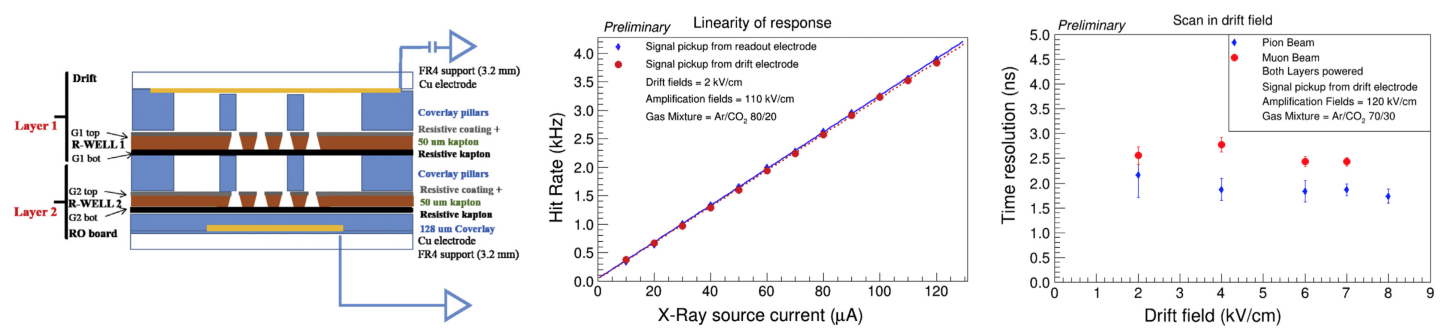

Figure 3: Transverse view of the first prototype of FTM detector (left). Measured hit rate from both channels of the FTM detector as a function of the X-ray current, which is proportional to the incident flux, up to the maximum available from the source (center). Measured time resolution for different values of applied drift field: the red points refer to muon beam, the blue diamonds to pion beam (right).

\section{Acknowledgments}

We gratefully acknowledge the support of FRS-FNRS (Belgium), FWO-Flanders (Belgium), BSF-MES (Bulgaria), BMBF (Germany), DAE (India), DST (India), INFN (Italy), NRF (Korea), QNRF (Qatar), and DOE (USA).

\section{References}

[1] L. Evans and P. Bryant, LHC Machine, 3 (2008) S08001.

[2] CMS Collaboration, The CMS experiment at the CERN LHC, 3 (2008) S08004.

[3] F. Sauli, GEM: A new concept for electron amplification in gas detectors, Nucl. Instrum. Meth. A386 (1997) 531.

[4] De Oliveira, Rui and Maggi, Marcello and Sharma, Archana, A novel fast timing micropattern gaseous detector: FTM, CERN-OPEN-2015-002-INFN-15-01-BA

[5] CMS Collaboration, CMS Technical Design Report for the Muon Endcap GEM Upgrade, CERN-LHCC-2015-012, CMS-TDR-013

[6] CMS Collaboration, Technical Proposal for the Phase-II Upgrade of the CMS Detector, CERN-LHCC-2015-010, LHCC-P-008, CMS-TDR-15-02

[7] GEM Collaboration, $R \& D$ on a new type of micropattern gaseous detector: The Fast Timing Micropattern detector, Nuclear Instruments and Methods in Physics Research Section A, 0168-9002, http://dx.doi.org/10.1016/j.nima.2016.05.067 